\title{
Online Detection of Fire in Video
}

\author{
B. Uğur Töreyin, A. Enis Çetin \\ Bilkent University 06800 Ankara Turkey \\ \{ugur, cetin\}@ee.bilkent.edu.tr
}

\begin{abstract}
This paper describes an online learning based method to detect flames in video by processing the data generated by an ordinary camera monitoring a scene. Our fire detection method consists of weak classifiers based on temporal and spatial modeling of flames. Markov models representing the flame and flame colored ordinary moving objects are used to distinguish temporal flame flicker process from motion of flame colored moving objects. Boundary of flames are represented in wavelet domain and high frequency nature of the boundaries of fire regions is also used as a clue to model the flame flicker spatially. Results from temporal and spatial weak classifiers based on flame flicker and irregularity of the flame region boundaries are updated online to reach a final decision. False alarms due to ordinary and periodic motion of flame colored moving objects are greatly reduced when compared to the existing video based fire detection systems.
\end{abstract}

\section{Introduction}

Conventional point smoke and fire detectors typically detect the presence of certain particles generated by smoke and fire by ionisation or photometry. An important weakness of point detectors is that they are distance limited and fail in open or large spaces. The strength of using video in fire detection is the ability to monitor large and open spaces. Earlier fire and flame detection algorithms are based on the use of color and motion information in video [10]. There are also some recent papers proposing methods which characterize the specific motion of flames [12],[17],[5],[18],[20]. Other recent methods for video based fire detection are [9],[21],[19]. These methods are developed to detect the presence of smoke in the video.

It is well-known that turbulent flames flicker with a frequency of around $10 \mathrm{~Hz}$ [1]. Therefore, fire detection scheme can be made more robust by detecting periodic high-frequency behavior in flame colored moving pixels compared to existing fire detection systems described in [12] and [5]. In practice, flame flicker frequency is not constant and it varies in time. In fact, variations in flame pixels can be considered as random events. Therefore, a Markov model based modeling of flame flicker process produces more robust performance compared to frequency domain based methods.

If the contours of an object exhibit rapid time-varying behavior then this is an important sign of presence of flames in the scene. This time-varying behavior is directly observable in the variations of color channel values of the pixels under consideration. Hence, Markov models corresponding to flame and non-flame pixels are built as consisting of states representing relative locations of the pixels in the color space. In addition, boundaries of flame colored moving regions are estimated in each image frame. A onedimensional curve (1-D) representing the distance to the boundary from the center of mass of the region is extracted for each of those regions. The wavelet transform of this 1-D curve is computed and the high frequency nature of the contour of the fire region is determined using the energy of the wavelet signal. This spatial domain clue is also combined with temporal clues to reach a decision.

Online training is especially important for video based fire detection applications to adapt to the environment that the system is installed. It is impossible to train the fire detection system for every possible scenario whenever the system is installed to a new location. There are various online learning algorithms in the literature including online bagging and boosting [16], online Arc- $\mathrm{x} 4$ [7] and weighted majority algorithm [13]. In this paper, we use weighted majority algorithm to detect flames in video by combining the results from the weak classifiers based on flame flicker and irregularity of the flame region boundaries.

\section{Markov Model Based Modeling of Flame Flicker}

Methods of identifying flame in video include [10] and [12]. The method in [10] makes use of only the color information. On the other hand, the scheme in [12] is based on detecting the fire colored regions in the current video first. If these fire colored regions move then they are marked as possible regions of fire in the scene monitored by a camera. 
In video, the appearance of an object in which the contours, chrominance or luminosity oscillate similar to online/off-line trained flame data, constitutes a sign of the possible presence of flames. By incorporating temporal analysis around object boundaries, one can reduce the false alarms which may be due to flame colored ordinary moving objects. Flicker of turbulent flames significantly increases frequency content around $10 \mathrm{~Hz}$ [17]. Actually, the fire behavior is a wide-band random activity below $15 \mathrm{~Hz}$ and a random process based modeling approach is naturally suited to characterize the rapid time-varying characteristic of flame boundaries. Broadbent and Huang et al. independently reported different flicker frequency distributions for various fuel types in [2] and [11]. In general, a pixel especially at the edge of a flame becomes part of the fire and disappears in the background several times in one second of a video at random. In other words, a pixel especially at the edge of a flame could appear and disappear several times in one second of a video in a random manner. This characteristic behavior is very well suited to be modeled as a Markov model. Markov models are extensively used in speech recognition systems and recently they have been used in computer vision applications [3].

In [14], the shape of fire regions are represented in Fourier domain. Since, Fourier Transform does not carry any time information, FFTs have to be computed in windows of data and temporal window size is very important for detection. If it is too long then one may not get enough peaks in the FFT data. If it is too short than one may completely miss cycles and therefore no peaks can be observed in the Fourier domain. Another problem is that, one may not detect periodicity in fast growing fires because the boundary of fire region simply grows in video. However in Markov model approach, the rapid time-varying characteristic of flame boundaries, is naturally captured. The Markov model based approach is similar to the one described in [18].

Three-state Markov models are trained for both flame and non-flame pixels to represent the temporal behavior (cf. Fig.1). These models are trained using a feature signal which is defined as follows: Let $R_{k}(n)$ be the red-channel value of the $k-t h$ pixel at frame $n$. The wavelet coefficients of $R_{k}$ are obtained by the filterbank structure shown in Fig.2.

Wavelet signals can easily reveal the random characteristic of a given signal which is an intrinsic nature of flame pixels. That is why the use of wavelets instead of actual pixel values lead to more robust detection of flames in video. Since, wavelet signals are high-pass filtered signals, slow variations in the original signal lead to zero-valued wavelet coefficients. Hence it is easier to set thresholds in the wavelet domain to distinguish slow varying signals from rapidly changing signals. Non-negative thresholds $T_{1}<T_{2}$ are introduced in wavelet domain to define the three states

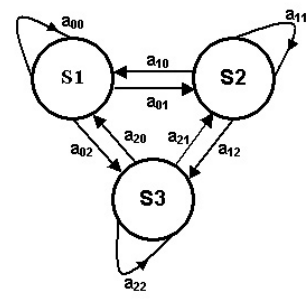

(a)

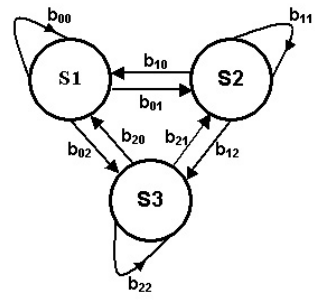

(b)
Figure 1. Three-state Markov models for a) flame and b) non-flame moving pixels.

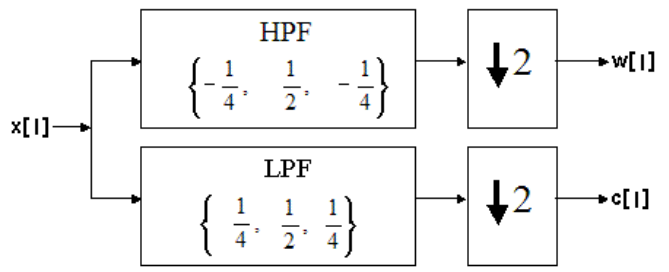

Figure 2. Single-stage wavelet filter bank. The high-pass and the low-pass filter coefficients are $\left\{-\frac{1}{4}, \frac{1}{2},-\frac{1}{4}\right\}$ and $\left\{\frac{1}{4}, \frac{1}{2}, \frac{1}{4}\right\}$, respectively.

of the Hidden Markov Models for flame and non-flame firecolored moving objects. The states of HMMs are defined as follows: at time $n$, if $|w(n)|<T_{1}$, the state is in $S 1$; if $T_{1}<|w(n)|<T_{2}$, the state is $S 2$; else if $|w(n)|>T_{2}$, the state $S 3$ is attained. For the pixels of regular fire-colored objects like walking people with reddish shirts, a red moving car, etc., no rapid changes take place in the pixel values. Therefore, the temporal wavelet coefficients ideally should be zero but due to noise the wavelet coefficients wiggle around zero. The lower threshold $T_{1}$ basically determines a given wavelet coefficient being close to zero. The second threshold $T_{2}$ indicates that the wavelet coefficient is significantly higher than zero. When the wavelet coefficients fluctuate between values above the higher threshold $T_{2}$ and below the lower threshold $T_{1}$ in a frequent manner this indicates the existence of flames in the viewing range of the camera.

The transition probabilities between states for a pixel are estimated during a pre-determined period of time around flame boundaries. During the recognition phase, the HMM based analysis is carried out in pixels near the contour boundaries of fire-colored moving regions. The state sequence of length 20 image frames is determined for these candidate pixels and fed to the flame and non-flame pixel models. The model yielding higher probability is determined as the result of the analysis for each of the candidate pixel. A pixel is called as a flame or a non-flame pixel according to the result of this analysis. This is the temporal weak classifier to be used in online detection/training 
framework.

\section{Wavelet Domain Analysis of Moving Object Contours}

Moving objects in video are detected using the background estimation method developed by Collins et al. [6]. This method assumes that the camera is stationary. Moving pixels are determined by subtracting the current image from the background image and thresholding. A recursive adaptive threshold estimation is described in [6] as well. Other methods can be also used for moving object estimation. After moving object detection, it is checked whether the pixels of the moving object satisfy the color conditions typical for flames.

The flame color model of [5] is used for defining the flame-pixels. Although there are various types of fires, flames especially in the initial stages of the fire exhibit a color range of red to yellow. In terms of $R G B$ values, this fact corresponds to the following inter-relation between $R, G$ and $B$ color channels: $R>G$ and $G>B$. The combined condition for the fire region in the captured image is $R>G>B$. Besides, $R$ should be more stressed than the other components, because $R$ becomes the dominating color channel in an $R G B$ image of flames. This imposes another condition for $R$ as to be over some predetermined threshold, $R_{T}$. However, lighting conditions in the background may adversely affect the saturation values of flames resulting in similar $R, G$ and $B$ values which may cause non-flame pixels to be considered as flame colored. Therefore, saturation values of the pixels under consideration should also be over some threshold value.

The next step of the proposed method is to determine the center of mass of the moving fire-colored object. A onedimensional (1-D) signal $x(\theta)$ is obtained by computing the distance from the center of mass of the object to the object boundary for $0 \leq \theta<2 \pi$. In Fig.3, two image frames are shown. Example feature functions for the fire-colored moving car and the fire region in Fig. 3 are shown in Fig. 4 for 64 equally spaced angles $x[l]=x\left(l \theta_{s}\right), \theta_{s}=\frac{2 \pi}{64}$. To determine the high-frequency content of a curve, we use the same single scale wavelet transform as shown in Fig. 2. The feature signal $x[l]$ is fed to the filterbank shown in Fig. 2 and the low-band signal

$$
c[l]=\sum_{m} h[2 l-m] x[m]
$$

and the high-band subsignal

$$
w[l]=\sum_{m} g[2 l-m] x[m]
$$

are obtained. Coefficients of the lowpass and the highpass filters are $h[l]=\left\{\frac{1}{4}, \frac{1}{2}, \frac{1}{4}\right\}$ and $g[l]=\left\{-\frac{1}{4}, \frac{1}{2},-\frac{1}{4}\right\}$, respectively [8], [4].
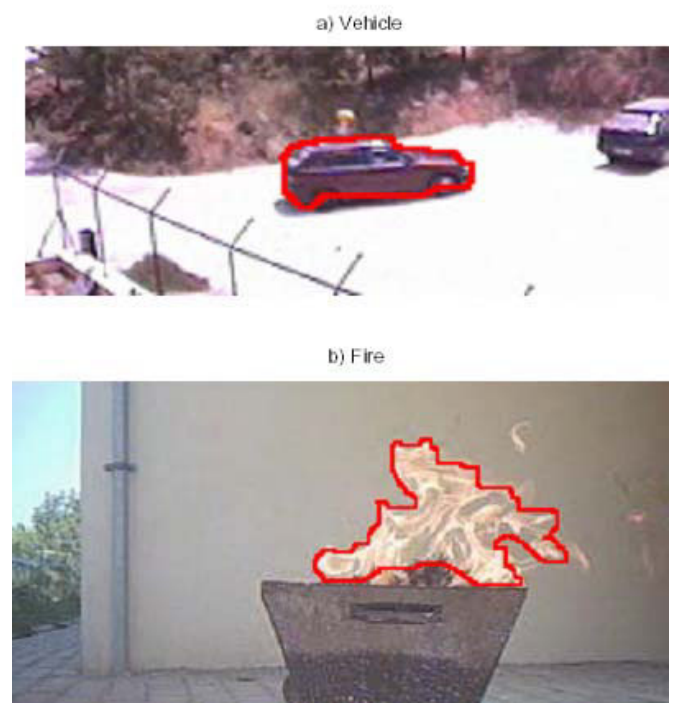

Figure 3. Two fire-colored moving objects in video: a) a vehicle, and b) fire image. Moving objects are determined by the hybrid background subtraction algorithm of [6].

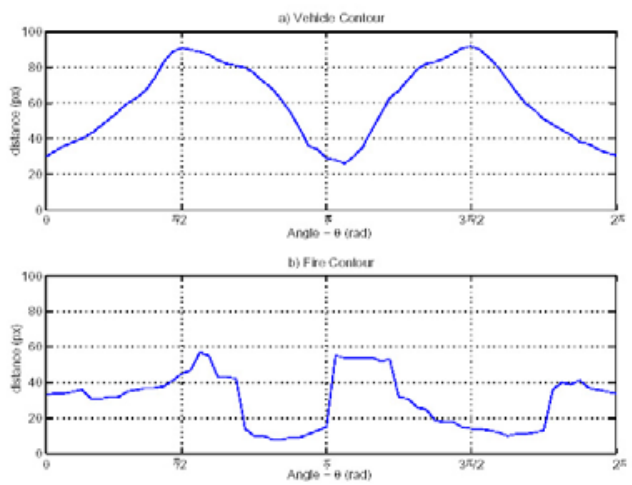

Figure 4. Equally spaced 64 contour points of the a) the vehicle, and b) the fire regions shown in Fig.3.

The absolute values of wavelet, $w[l]$ and low-band $c[l]$ coefficients of the fire region and the car are shown in Fig. 5 and Fig.6, respectively. The high-frequency variations of the feature signal of the fire region is clearly distinct from that of the car. Since regular objects have relatively smooth boundaries compared to flames, the high-frequency wavelet coefficients of flame boundary feature signals have more energy than regular objects. Therefore, the ratio of the wavelet domain energy to the energy of the low-band signal is a good indicator of a fire region. This ratio is defined as

$$
\rho=\frac{\sum_{l}|w[l]|}{\sum_{l}|c[l]|}
$$

The likelihood of the moving region to be a fire region is highly correlated with the parameter $\rho$. Higher the value of 

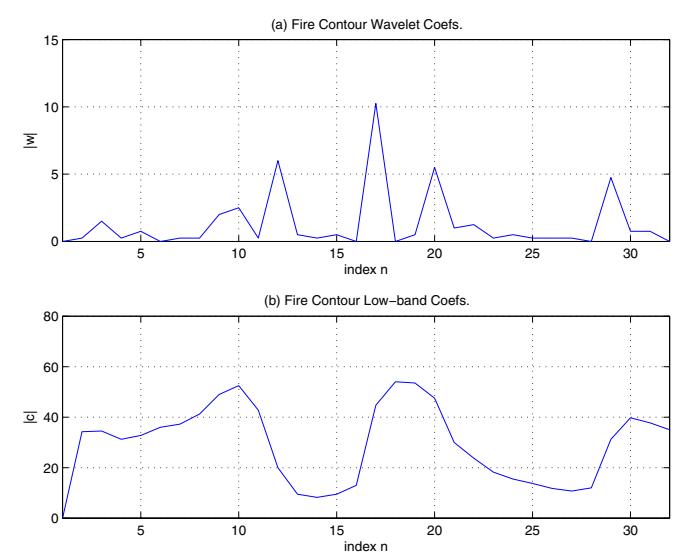

Figure 5. The absolute values of a)high-band (wavelet) and b)lowband coefficients for the fire region.
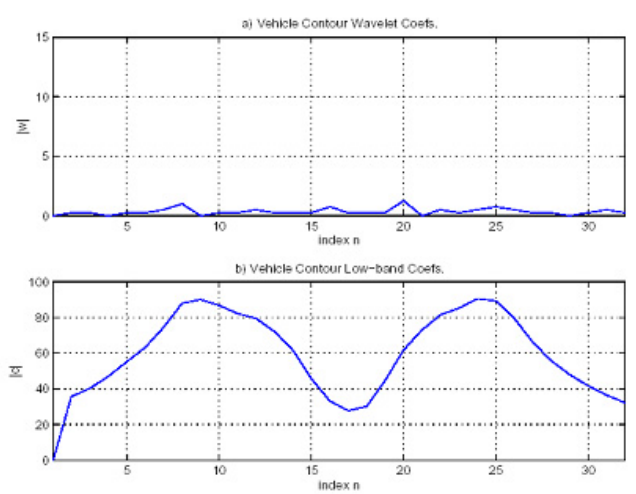

Figure 6. The absolute values of a)high-band (wavelet) and b)lowband coefficients for the car region.

$\rho$, higher the probability of the region belonging to flame regions.

Initially, a threshold $\rho_{T}$ for $\rho$ is experimentally estimated. During real-time analysis, regions for which $\rho>\rho_{T}$ are determined. Such regions are possible fire regions. In order not to miss any fire region, a low threshold value for $\rho_{T}$ is selected initially. This threshold is updated online after installing the system to a specific location as described in the next section.

\section{Weighted Majority Based Online Training}

We use a weighted majority based online training method to adapt the fire detection system to varying conditions in the environment. Weighted Majority algorithm assigns weights on several weak classifiers and increase or decrease their weights depending on whether the individual classifiers correctly classify or misclassify the training example currently being considered, respectively [15]. The algorithm is presented in Fig.7.

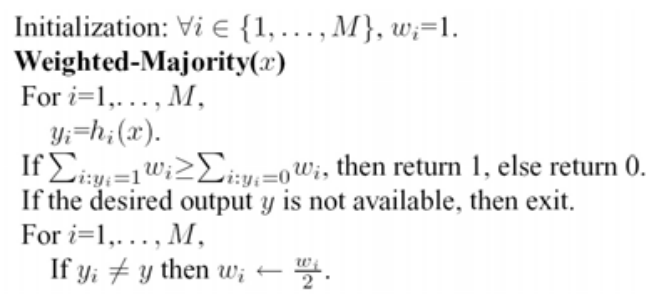

Figure 7. Weighted majority algorithm used in fire detection system. Weights, $w_{i}$ 's, are updated for each of the $M$-many weak classifiers, $h_{i}$ 's, whenever a new training data, $x$, arrives. The correct classification value of the data $x$ is $y ; y_{i}$ is the classification result of the $i-t h$ weak classifier, $h_{i}$.

Markov model based temporal classifiers described in Sec. 2 for every fire-colored moving pixel and contour based classifier explained in Sec.3 for every fire-colored moving region in an image frame are considered as separate weak classifiers, $h_{i}$ 's, in our online learning framework. They are called as temporal and spatial weak classifiers, respectively. An output class value, being either a fire, 1 , or a non-fire, 0 , is assigned to each and every one of these weak classifiers in 20 frame long periods as separate decisions. According to their individual success in classification, i.e. whether the value of $y_{i}$ is equal to the value of $y$ or not, weights corresponding to these weak classifiers are updated by weighted majority algorithm.

Apart from weight updates, for the $i-t h$ spatial weak classifier, $h_{i}$, when $y_{i} \neq y$, the threshold value $\rho_{T}$ is updated according to the following rule:

$$
\rho_{T} \leftarrow \begin{cases}\frac{\rho_{T}}{2} & \text { if } \rho<\rho_{T} \\ 2 \rho_{T} & \text { otherwise }\end{cases}
$$

This way, the system 'learns' the environment and adapts itself online to variations in the characteristics of the incoming data.

\section{Experimental Results}

The proposed method was implemented in a personal computer with an AMD AthlonXP 2000+ 1.66GHz processor and tested for a large variety of conditions in comparison with the method in [18] which fuses the individual decisions from weak classifiers by a simple 'and' operation. The method in [18] reports false alarms for a fire colored dancing man arbitrarily waving arms in order to fool the system. It also issues false alarms for moving fire-colored objects exhibiting periodic motion such as rotating ambulance lights in a tunnel and their reflections from the walls.

These false alarms are eliminated with the online learning based training approach. The system succeeds in adapting itself online. In addition, the proposed system issues fire alarms for all of the test video clips containing fire used 

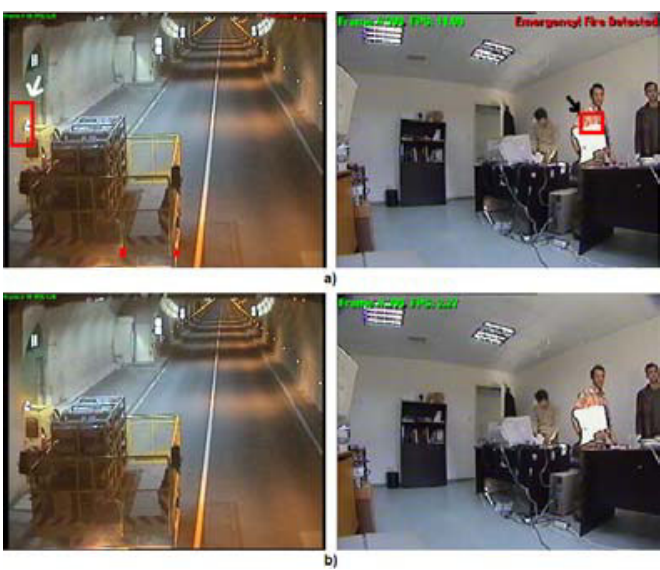

Figure 8. Detection results of a) the method in [18] and b) the proposed online learning method. False alarms are eliminated with the proposed method for periodically rotating fire-colored lights in a tunnel and fire-colored dancing man.

in [18]. Some comparative classification results are presented in Fig.8.

\section{Conclusion}

An online learning based method for fire detection is proposed. Color and regular motion clues are used to reduce the size of the search space. HMM based temporal flicker modeling of flames and wavelet based contour modeling approaches are used as weak classifiers. A weighted-majority based method is utilized for online learning. Experimental results show that false alarms issued by earlier methods can be drastically reduced by using separate Markov models for flame and non-flame moving pixels. The method can be used for automatically training the fire detection system when installed at a different location.

In [19], several weak smoke detectors are fused together with a simple logical 'and' operation. A similar online learning approach can be developed for the smoke detection method in [19], as well.

\section{References}

[1] B. W. Albers and A. K. Agrawal. Schlieren analysis of an oscillating gas-jet diffusion. Combustion and Flame, 119:8494, 1999. 1

[2] J. A. Broadbent. Fundamental flame flicker monitoring for power plant boilers. In IEE Seminar on Advanced Sensors and Instrumentation Systems for Combustion Processes, pages $4 / 1-4 / 4,2000.2$

[3] H. Bunke and T. C. (Eds.). HMMs Applications in Computer Vision. World Scientific, 2001. 2

[4] A. E. Cetin and R. Ansari. Signal recovery from wavelet transform maxima. IEEE Trans. on Signal Processing, 42:194-196, 1994. 3
[5] T. Chen, P. Wu, and Y. Chiou. An early fire-detection method based on image processing. In ICIP '04, pages 1707-1710, 2004. 1, 3

[6] R. T. Collins, A. J. Lipton, and T. Kanade. A system for video surveillance and monitoring. In $8^{\text {th }}$ Int. Topical Meeting on Robotics and Remote Systems. American Nuclear Society, 1999. 3

[7] A. Fern and R. Givan. Online ensemble learning: An empirical study. Machine Learning, 53(1-2):71-109, 2003. 1

[8] O. N. Gerek and A. E. Cetin. Adaptive polyphase subband decomposition structures for image compression. IEEE Transactions on Image Processing, 9:1649-1659, October 2000. 3

[9] P. Guillemant and J. Vicente. Real-time identification of smoke images by clustering motions on a fractal curve with a temporal embedding method. Optical Engineering, 40(4):554-563, 2001. 1

[10] G. Healey, D. Slater, T. Lin, B. Drda, and A. D. Goedeke. A system for real-time fire detection. In $C V P R$ '93, pages 15-17, 1993. 1

[11] H. Huang, Y. Yan, G. Lu, and A. Reed. On-line flicker measurement of gaseous flames by imaging processing and spectral analysis. Measurement Science and Technology, 10:726733, 1999. 2

[12] W. P. III, M. Shah, and N. V. Lobo. Flame recognition in video. Pattern Recognition Letters, 23(1-3):319-327, 2002. 1

[13] N. Littlestone and M. Warmuth. The weighted majority algorithm. Information and Computation, 108:212-261, 1994. 1

[14] C. B. Liu and N. Ahuja. Vision based fire detection. In ICPR '04, volume 4, 2004. 2

[15] N. Oza. Online ensemble learning. PhD Dissertation, 2002. 4

[16] N. Oza and S. Russell. Online bagging and boosting. In Artifical Intelligence and Statistics, pages 105-112, 2001. 1

[17] F. T. SA. Method and Device for Detecting Fires Based on Image Analysis. PCT Pubn.No: WO02/069292, Boulevard de Grancy 19A, CH-1006 Lausanne, Switzerland, 2002. 1, 2

[18] B. U. Toreyin, Y. Dedeoglu, and A. E. Cetin. Flame detection in video using hidden markov models. In ICIP '05, pages 1230-1233, 2005. 1, 2, 4, 5

[19] B. U. Toreyin, Y. Dedeoglu, and A. E. Cetin. Wavelet based real-time smoke detection in video. In $13^{\text {th }}$ European Signal Processing Conference EUSIPCO, 2005. 1, 5

[20] B. U. Toreyin, Y. Dedeoglu, U. Gudukbay, and A. E. Cetin. Computer vision based system for real-time fire and flame detection. Pattern Recognition Letters, 27:49-58, 2006. 1

[21] J. Vicente and P. Guillemant. An image processing technique for automatically detecting forest fire. International Journal of Thermal Sciences, 41:1113-1120, 2002. 1 